\title{
UM SIMULADOR DE CASOS CLÍNICOS COMPLEXOS NO PROCESSO DE APRENDIZAGEM EM SAÚDE
}

\author{
Paulo Ricardo Muniz Barros - UFCSPA - pbarros1979@gmail.com \\ Sílvio César Cazella - UFCSPA/UNISINOS - silvio.cazella@ gmail.com \\ Marta Bez - UFRGS/FEVALE - martabez@gmail.com \\ Cecília Dias Flores - UFCSPA - dflores@ufcspa.edu.br \\ Alessandra Dahmer - UFCSPA - adahmer@ufcspa.edu.br \\ João Batista Mossmann - FEVALE - mossmann@feevale.br \\ João Marcelo Fonseca - UFCSPA - joaomarcelofonseca@gmail.com \\ Vinícius Maroni - UFCSPA - viniciusmaroni@gmail.com
}

\begin{abstract}
Resumo. Este artigo apresenta o projeto SimDeCS, que representa uma importante oportunidade para estabelecer uma nova forma de relação entre educador e educando, inserindo ferramentas informatizadas na forma de simuladores de casos clínicos complexos no processo de aprendizado. A experiência tem como objetivo apresentar um processo mais atrativo $e$ próximo das situações do mundo real reduzindo as distâncias entre a teoria e a prática clínica, através da utilização de simulações. $O$ sistema encontra-se em fase de prototipação, porém os primeiros testes realizados mostraram-se bastante promissores.
\end{abstract}

Palavras-chaves: Educação Médica, Simulação, Casos Clínicos.

\section{A SIMULATOR OF CLINICAL COMPLEX CASES IN THE PROCESS OF LEARNING IN HEALTH}

\begin{abstract}
This paper presents the SimDeCS project, which represents an important opportunity to establish a new form of relationship between teacher and student, introducing computerized tools to simulate complex clinical cases in the learning process. The experience aims to present a more attractive and effective learning process by reducing the gap between theory and clinical practice through the use of simulations. The system is in prototype phase, but the preliminary tests have presented very promising.
\end{abstract}

Keywords: Medical Education, Simulation, Case Studies.

\section{Introdução}

A formação e a capacitação de recursos humanos em saúde devem estar associadas às adequações dos modos de atenção à saúde, criando-se, dessa forma, os cenários propícios à aprendizagem e à organização da cadeia do cuidado em saúde. Neste contexto, entendem-se os cenários de aprendizagem como a incorporação e a interseção 
de métodos didático-pedagógicos; de áreas de práticas e vivências; de utilização de tecnologias e habilidades cognitivas e psicomotoras; de valorização dos preceitos morais e éticos, orientadores de condutas individuais e coletivas; de organização do processo de trabalho (MARIS, 2004).

A imposição desta realidade sugere a necessidade de reformulação do ensino, buscando a inclusão de recursos tecnológicos na formação destes educandos. A área da saúde (no que tange ao diagnóstico investigativo e a conduta terapêutica) caracteriza-se como um ambiente de domínio incerto. Nestes ambientes destaca-se como alternativa de ensino a utilização de simuladores. Os simuladores de casos clínicos são considerados ferramentas muito poderosas na educação de profissionais de saúde, de acordo com a Organização Pan-Americana de Saúde ${ }^{1}$.

As simulações podem ser representadas de diversas formas, entre elas através de jogos computacionais aplicados ao ensino, conhecidos como jogos sérios (Serious Games). Sua principal característica é ensinar conteúdos específicos de disciplinas ou treinar habilidades tanto operacionais como comportamentais (MORAIS, 2010).

Este artigo apresenta o projeto SimDeCS (Simulador de Casos de Saúde), que se constitui como uma importante oportunidade para estabelecer uma nova forma de relação entre educador e educando, inserindo ferramentas informatizadas na forma de simuladores de casos clínicos complexos no processo de aprendizado. Deste modo, caracteriza um processo mais atrativo e próximo das situações do mundo real, reduzindo as distâncias entre a teoria e a prática clínica.

Este artigo encontra-se dividido da seguinte forma: na seção 2 são apresentados os trabalhos relacionados à área de simulação, a seção 3 descreve questões relacionadas a modelagem de conhecimento. A seção 4 apresenta o SimDeCS implementado para simulação de casos clínicos focado na educação, a seção 5 descreve o processo de avaliação, e por fim, a seção 6 apresenta as conclusões.

\section{Trabalhos Relacionados}

A partir da idéia de que a aprendizagem representa uma mudança de comportamento, (GIUSTA, 1985), buscam-se formas de prover experiência a alunos. Nesta busca pelo conhecimento os simuladores têm se apresentado como grandes aliados, evoluindo e atraindo estudantes das mais variadas áreas do conhecimento. Busca-se, normalmente, nestas simulações, prover informações com características da vida real, permitindo a participação em cenários e situações próximas as vivenciadas no dia a dia.

Atualmente, não existem definições precisas do termo simulação. Para Machado, Moraes e Nunes (2009), as simulações visam apresentar situações práticas vivenciadas no dia-a-dia e tem o objetivo principal de proporcionar o treinamento de profissionais, em várias áreas do conhecimento, situações críticas, conscientização de crianças, jovens e adultos. O autor Ziv (2005) define simulação como uma "técnica em que se utiliza um simulador, considerando-se simulador como um objeto ou representação parcial ou total de uma tarefa a ser replicada".

\footnotetext{
${ }^{1}$ Ref: Relatório Técnico 02 / BR CNT 0900487.001
}

V. $10 \mathrm{~N}^{\mathrm{o}} 1$, julho, 2012 
É possível encontrar fortes evidencias na literatura de que a simulação computacional pode melhorar a forma de conduzir o ensino, principalmente em casos onde há riscos ou em que seja impossível demonstrar diretamente um fenômeno (AKPAN, 2001). O uso de simulação computacional traz como vantagens a redução de tempo desprendido nos experimentos; manipulação fácil de variáveis para observação de várias hipóteses; a existência de recursos multivariados para a visualização dos resultados; a possibilidade de um aluno de medicina ver situações e tipos de pacientes que dificilmente seria possível em acompanhamentos reais (BLAKE and SCANLON, 2007; STANFORD, 2010). Conforme Stanford (2010) o tempo necessário para criar e configurar o ambiente da simulação é ainda uma desvantagem a ser superada.

O uso de simulação permite levar novas abordagens para o ensino e para a prática médica, denominadas de Medicina Baseado em Simulação. Para fins de treinamento, os simuladores podem ser aplicados para apresentar situações críticas que envolvam algum tipo de risco, tomada de decisões ou desenvolver habilidades específicas. Com base nisso, os simuladores para a área médica tem evoluído muito.

$\mathrm{Na}$ área da saúde, há exemplos de diversos tipos de simuladores. Na tabela 1 , são apresentados alguns exemplos retirados da literatura:

Tabela 1. Exemplos de Simuladores

\begin{tabular}{|c|c|c|}
\hline Autor & Ano & Foco de estudo \\
\hline Rendas et al & 1999 & PBLS - Framework to PBL problems to pathophysiology. \\
\hline Bergin and Fors & 2003 & ISP - Interactive simulator patient. \\
\hline Kneebone & 2003 & $\begin{array}{l}\text { Surgical training (vascular acess, sigmoidoscopy and } \\
\text { anastomosis). }\end{array}$ \\
\hline $\begin{array}{l}\text { Machado, L. S. et } \\
\text { al }\end{array}$ & 2003 & Oncologia pediátrica em 3D. \\
\hline $\begin{array}{l}\text { Holzinger, } \\
\text { Emberger et al }\end{array}$ & 2008 & $\begin{array}{l}\text { Cytogenetics, as an integral part of diagnostics in dysmorphology, } \\
\text { syndromology, prenatal and developmental diagnosis, reproductive } \\
\text { medicine, neuropediatrics, hematology and oncology. }\end{array}$ \\
\hline Smith and Roehrs & 2009 & $\begin{array}{l}\text { Patient with respiratory disorder who was going into respirator } \\
\text { distress. }\end{array}$ \\
\hline Ying & 2009 & A respiratory patient who was admitted for a pleural effusion. \\
\hline Holzinger et al & 2009 & $\begin{array}{l}\text { HAEMOSIM - Haemodynamics Simulator: physiological models } \\
\text { and the dynamics of complex mathematical rules related to these } \\
\text { models. }\end{array}$ \\
\hline Botezatu et al & 2010 & VPS - Virtual Patient Simulation System. \\
\hline
\end{tabular}

Fonte: Elaborado pelos autores

\section{Modelagem do Conhecimento}


A modelagem do conhecimento, no simulador proposto, será feita por especialistas, amparados pelas diretrizes clínicas. Os componentes do raciocínio clínico serão representados por Redes Bayesianas.

Redes Bayesianas são uma das técnicas de inteligência artificial para representação do conhecimento incerto (PEARL, 1986). O conhecimento incerto é a presença da incerteza na base de conhecimentos a ser utilizada. Em geral, está associado a graus variáveis de probabilidades e o encadeamento e influência entre elas. O motor de inferência aplicado as redes Bayesianas calculam as probabilidades conjuntas de um grande número de variáveis.

$\mathrm{Na}$ área das Ciências da Saúde em geral e especificamente na área médica, decisões são muito freqüentemente tomadas a partir de cenários e da interpretação de probabilidades em conjunto (BLEAKLEY, BLIGH, BRICE, 2011). O Teorema de Bayes é a forma matemática de relacionar probabilidades encadeadas. Ele demonstra como alterar probabilidades considerando novas evidências, e assim, obtendo novas probabilidades. O Teorema de Bayes é a base da Inferência Bayesiana, sendo desta forma o cerne da tomada de decisão dentro da mesma.

Redes Bayesianas são representadas graficamente por grafos dirigidos acíclicos onde cada variável que se acredita ter influência é representada como um nodo. Cada nodo da rede é associado a uma tabela de probabilidades. Esta tabela é conhecida como Tabela de Probabilidades Condicionais, considerando que ela representa probabilidades de um evento ocorrer, dado a ocorrência ou não de seus pais. Caso um nodo não possua pais, dizemos que ele tem uma Tabela de Probabilidades Inicias. Além dessa representação numérica, o relacionamento qualitativo entre as variáveis é representado nas relações de dependência estabelecidas entre os nodos (PEARL, 1993).

Trabalhando com modelos gráficos na representação do conhecimento, torna-se mais intuitivo o entendimento dos mesmos. Segundo Castillo et al (CASTILLO, 1998), a representação gráfica de modelos probabilísticos apresenta explicitamente a relação entre as mesmas, além de conservar essas relações de forma qualitativa.

\subsection{Modelagem do Conhecimento pelo Especialista}

A primeira etapa da modelagem consiste em delimitar o conhecimento a ser representado. É freqüente nas Ciências da Saúde que um determinado problema a ser abordado seja ampliado em abrangência na medida em que novas variáveis e situações vão sendo conectadas, indefinidamente. Para evitar essa impossibilidade de representação, o especialista de domínio deve ter claro quais limites usará na representação do problema. Uma diretriz clínica pode ser um exemplo de fonte inicial para essa delimitação. Diretrizes clínicas constituem-se em posicionamentos ou recomendações (statements) sistematicamente desenvolvidas para orientar médicos e pacientes acerca de cuidados de saúde, apropriados em circunstâncias clínicas específicas (PORTELA, 2008 apud IOM, 1990).

A etapa seguinte consiste em elencar o máximo de variáveis que possam estar envolvidas no problema. Para cada uma dessas variáveis deverá ser atribuída uma probabilidade inicial. Essa probabilidade poderá advir da própria fonte do conhecimento (diretriz clínica, levantamento epidemiológico, casuística disponível) ou, na ausência 
destes, de uma estimativa da prevalência na população que está sendo representada no problema.

A terceira etapa consiste em estabelecer as ligações conhecidas (qualitativas) entre os diferentes nodos. É essencial não haver ciclos, pela natureza da aplicação do Teorema de Bayes, de forma que as probabilidades ao se propagarem na rede não se retroalimentem.

A última etapa consiste na avaliação da plausibilidade clínica da rede constituída, com a possibilidade de calibração das probabilidades iniciais para um melhor desempenho no julgamento do especialista de domínio.

A representação do conhecimento é criada por especialistas, modeladas em uma RB, que, conforme Schwartz e Elstein (2008), se justifica em função de revisões na literatura médica que indicam que o processo de decisão e raciocínio diagnóstico é incerto, muitas vezes com informações imprecisas.

$\mathrm{Na}$ construção do caso de estudo, ao incluir sinais e sintomas disponíveis na rede, o professor propaga as probabilidades, emergindo então, um ou mais diagnósticos e suas consequentes condutas, modelando assim o caso que será simulado pelos alunos.

Os casos clínicos criados pelos professores ficam armazenados em um Banco de Dados, contendo os nodos selecionados pelo professor para as etapas de investigação, diagnóstico e conduta. Nele também são armazenadas informações adicionais sobre o caso clínico, bem como dados da ficha (ou prontuário) do paciente. Os nodos da rede que representam a investigação são armazenados no formato de perguntas, disponíveis no simulador para que, na execução, o aluno possa investigar o caso clínico. A cada pergunta realizada pelo aluno ao simulador, é consultada a rede modelada pelo professor e obtida uma resposta (simulando a resposta de um paciente em consulta) que expressa a probabilidade do nodo naquele momento.

\subsection{Exemplo de Rede}

Na figura 1, é apresentado um fragmento de rede que representa o conhecimento de um caso clínico de estudo. A partir dos sintomas (tosse, febre e emagrecimento) é representada a relação destes com a investigação usual (fibrobroncoscopia, pesquisa de Bacilos Álcool-Ácido Resistentes, Bacteriológico de escarro, Raio X de tórax).

Conforme esses resultados, emergem da rede as probabilidades para os diagnósticos de tuberculose pulmonar ou pneumonia, sendo esta última vinculada ao tratamento medicamentoso pertinente. 


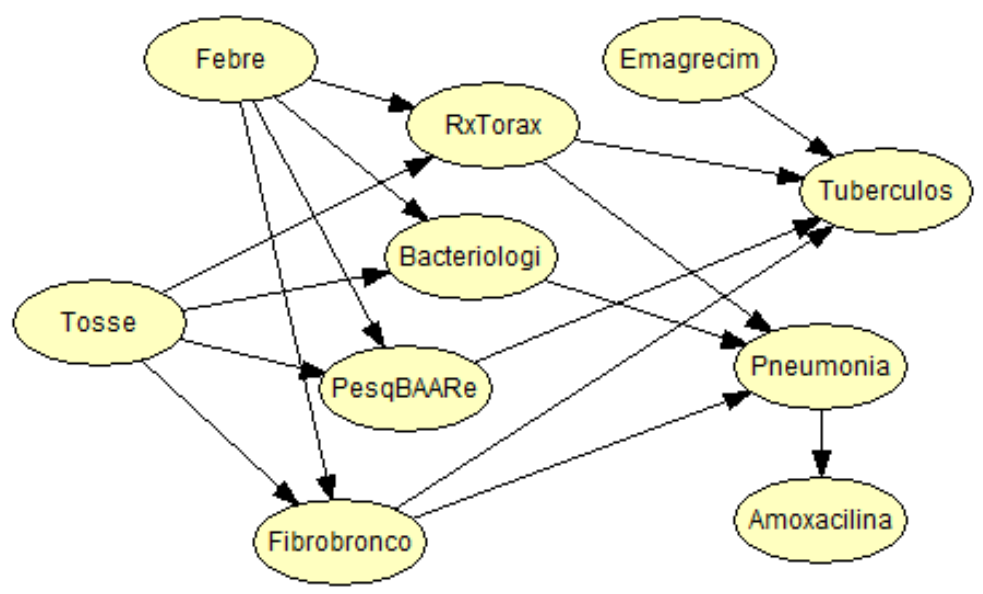

Figura 1. Exemplo de Rede Bayesiana 


\section{Simulador SimDeCs}

No simulador, com a interface modelada em 3D (figura 2), o aluno interage com o ambiente utilizando o mouse. Quando o aluno pretender interagir com um paciente, ele deve clicar sobre a imagem do paciente, o que lhe dará acesso a informações como idade, peso, altura e nome (propriedades do paciente). Além dessas informações, o ambiente apresenta ao aluno um menu que representa as características presentes no caso modelado pelo professor.

Para acessar estas características, que representam atividades possíveis no simulador, basta clicar sobre a opção desejada. Através da funcionalidade intitulada de "Realizar Exame Físico", por exemplo, o aluno terá acesso aos exames inseridos durante a modelagem do caso.

Caso escolha, por exemplo, "Exames Complementares", uma nova interface é apresentada ao usuário, permitindo a reprodução de imagens ou outras mídias, que contenham informações importantes para a simulação. No caso da Figura 3, é apresentada uma interface contendo uma radiografia, obtida a partir da opção "Solicitar Exame Físico".

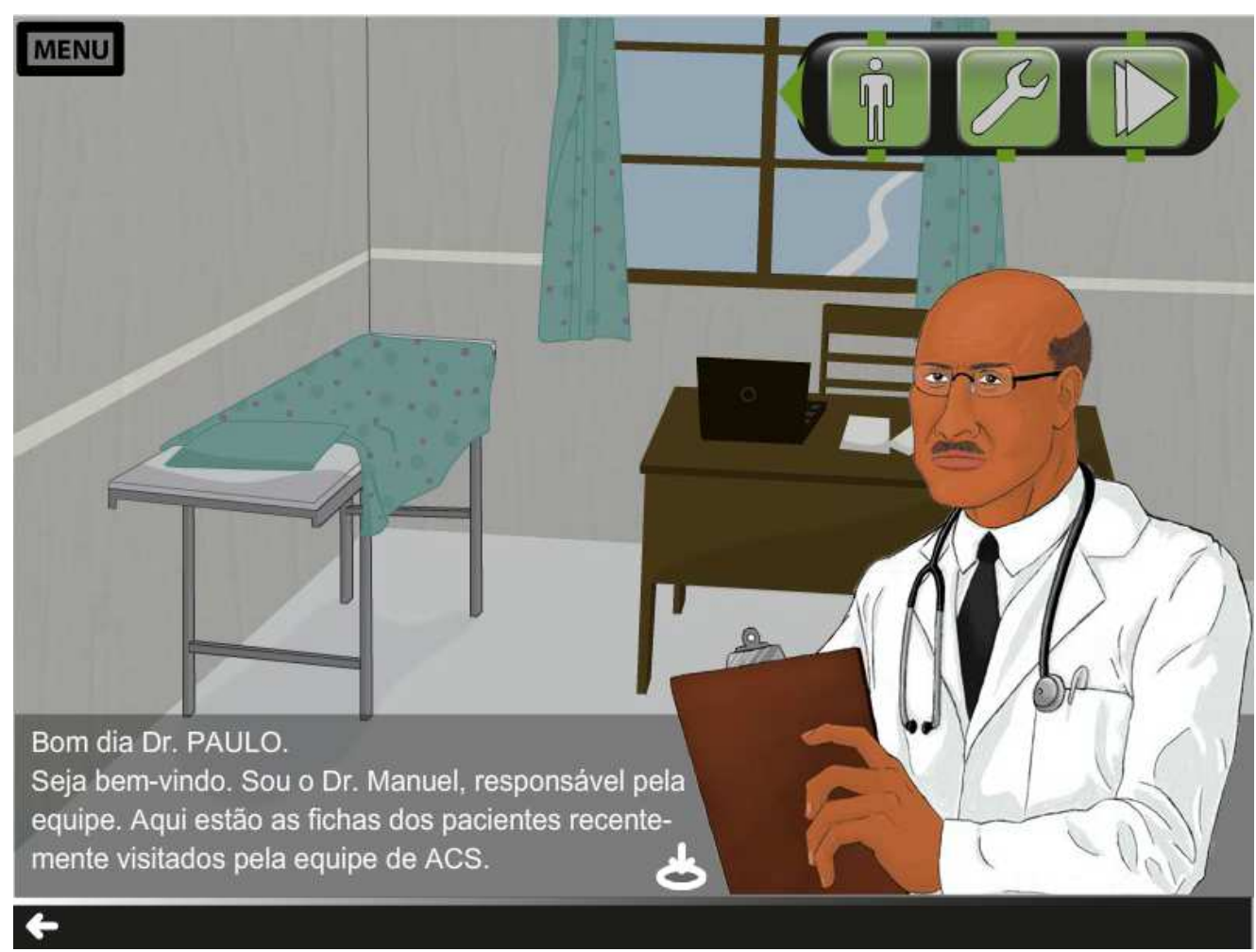

Figura 2. Simulação em 3D com atendimento ao paciente

A criação do player que possui a responsabilidade de executar as simulações construídas no ambiente é fundamental para o sucesso do simulador. Esse player constitui-se na parte onde a criatividade pode propiciar novas metáforas para que os 
alunos interajam na simulação e tenha acesso aos elementos do domínio representados pela rede bayesiana e banco de casos.

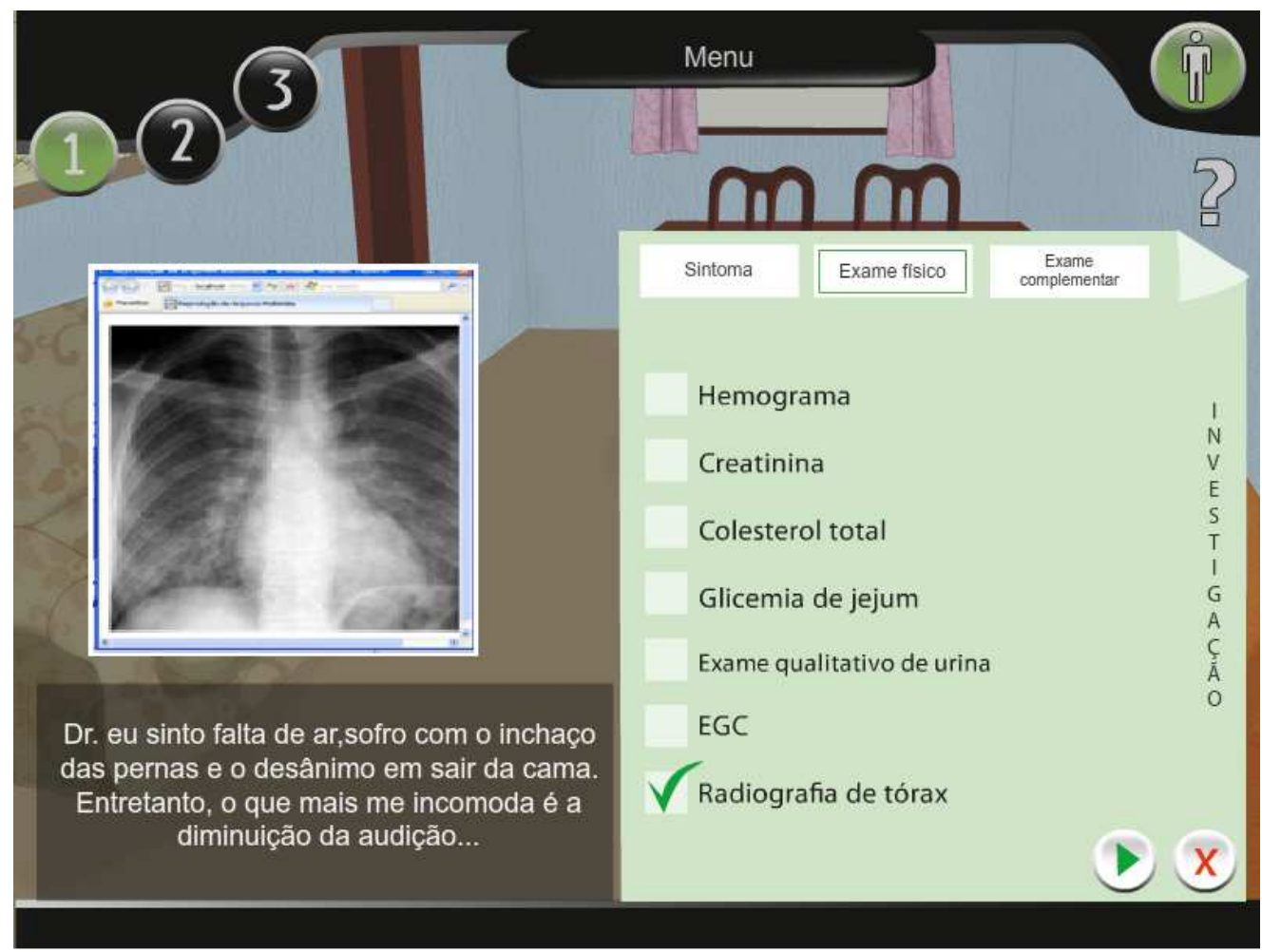

Figura 3 - Simulação em 3D com resultado de exame físico

\section{Avaliação}

Quando simuladores são aplicados a ambientes de ensino se torna fundamental estabelecer uma forma de avaliação dos educandos submetidos a estes ambientes. No caso específico de simulação de casos clínicos para a área da saúde, todo o trajeto percorrido pelo aluno durante a resolução do problema proposto deve ser considerado no momento de definição de um resultado, não ficando esta avaliação restrita somente ao resultado final encontrado.

Segundo SEIXAS; FLORES et. al. (2004), o ideal seria trabalharmos avaliando todas as hipóteses possíveis da construção do conhecimento, garantindo ao fim a compreensão por parte do educando do resultado encontrado. Evita-se assim, que o aluno faça sem compreender e estimulando-o a refletir sobre o que fazer.

Para suprir esta lacuna, entre o processo de construção do conhecimento e a chegada ao resultado final, utilizamos um motor de inferência para análise das decisões tomadas pelo aluno durante o processo de aprendizagem. Atribuindo assim valores mensuráveis quanto ao custo e a efetividade das escolhas e avaliando o tempo transcorrido até o diagnóstico; assim como o custo em exames complementares realizados questão relevante a medicina de família e comunidade.

Para construção deste motor de inferência dividimos o processo de avaliação, assim como a simulação, em três grandes etapas a saber: 
1. Investigação e construção da hipótese diagnóstica;

2. Seleção do diagnostico presuntivo para o caso;

3. Desfecho ou conduta terapêutica.

Na primeira fase o aluno investiga os pacientes participantes do caso através da observação de sinais, sintomas, exames físicos e complementares. Nesta busca, o simulador utiliza-se das informações de tempo, custo e influência no diagnostico dos nodos modelados na rede bayesiana para avaliar, além do diagnostico encontrado, também o caminho percorrido pelo aluno.

Nesta primeira fase, investigativa, alguns pontos fundamentais são considerados para obtenção do cálculo de desempenho do estudante. Ao diagnóstico presuntivo selecionado é atribuído um peso, sendo este influenciado pela complexidade de identificação do diagnóstico, ou seja, existe uma tolerância para casos clínicos que possibilitam mais de um diagnóstico plausível.

O tempo e o custo despendidos na busca de solução do caso são comparados com a rede modelada, desvios para baixo distanciando-se do esperado indicam um comportamento lotérico em que o discente pode ter chegado a uma conclusão baseada em poucas evidencias, portanto acarreta em uma redução de sua pontuação. Por outro lado, uma utilização demasiada do tempo e do custo também é penalizada, pois indica indecisão e desconhecimento do aprendiz submetido à simulação.

No momento em que o aluno chega a um diagnóstico presuntivo e seleciona a conduta terapêutica para o paciente, este passa a um segundo estágio da avaliação. Nesta fase, a adequação da conduta ao diagnóstico sugerido é avaliada, porém em casos de uma seleção diagnóstica equivocada, seguido de uma alternativa de tratamento adequada, tem uma intervenção diferente em relação ao erro completo de diagnóstico e tratamento.

O resultado da avaliação emerge na interface do simulador em pontos identificados como chave. Sendo eles disparados no momento em que diagnóstico presuntivo é selecionado ou quando a conduta terapêutica é determinada. Além disto, mensagens de apoio pedagógico e recondução podem emergir durante a investigação quando o agente mediador identificar que o aluno navega de forma cíclica pelas evidencias sem conseguir avançar no processo.

Ao final a avaliação do aluno é exibida na forma de um texto estruturado explicando os pontos onde o erro ou o sucesso ocorreu agindo como um tutor inteligente reforçando o aprendizado.

\section{Conclusão}

O SimDeCS torna-se um grande auxiliar no processo pedagógico de aprendizagem dos alunos seguindo as diretrizes do aprendizado baseado em problemas. No simulador o aprendiz é submetido a situações que se aproximam da realidade, podendo testar seus conhecimentos e ao fim ser avaliado, identificando seus acertos e falhas.

Atualmente, o SimDeCS encontra-se em fase de desenvolvimento, contando com um protótipo funcional que compreende a modelagem dos casos, algumas redes bayesianas e o ambiente do jogo em uma versão em três dimensões. 
Ao final desta fase, entende-se que o SimDeCS irá consolidar-se como uma valiosa ferramenta no processo de aprendizagem de alunos da área medica como já evidenciado em primeiros testes realizados.

Como trabalhos futuros verifica-se a finalização do protótipo, para validação do mesmo junto a alunos da área da saúde.

\section{Agradecimentos}

Agradecimento à CAPES, através do Projeto Pró-Ensino na Saúde e a Universidade Aberta do Sistema Único de Saúde (UNA-SUS).

\section{Referências}

AKPAN, J. P. (2001). Issues associated with inserting computer simulations into biology instruction: a review of the literature. Electronic Journal of Science Education, 5(3).

BERGIN, R.; YOUNGBLOOD, P.; AYERS, M. K.; BOBERG, J.; BOLANDER, K.; COURTEILLE, O.; DEV, p.; HINDBECK, H.; EDWARD, E.; STRINGER, J. R., THALME, A. FORS, U. G. H. (2003) Interactive Simulated Patient: Experiences with collaborative e-learning in medicine. Journal of Educational Computing Research. V.29, n.3, pp. 387-400

BLAKE, C.; SCANLON, E. (2007). Reconsidering simulations in science education at a distance: features of effective use. Journal of Computer Assisted Learning, 23(6), 491-502.

BLEAKLEY, A.; BLIGH, J.; BRICE, J. (2011). Medical Education for the Future. Springer. V. 1. 2011.

BOTEZATU, M., Hult, H; Fors, U. G. (2010). Virtual patient simulation: what do students make of it? A focus group study. BMC Medical Education, 2010 10:91.

CASTILlO, E.; GUTIÉRREZ, J. M.; HADI, A. S. (1998). Sistemas Expertos e Modelos de Redes Probabilisticas. [S.1.]: Academia Espanola de Ingenieria, 1998.

GIUSTA, A. da S. (1985) Concepções de Aprendizagem e Práticas Pedagógicas. In: Educ.Rev. Belo Horizonte, 1985. v.1: 24-31p

HOLZINGER, A.; Emberger, W.; Wassertheurer, S.; Neal, L. (2008). Design, development and evaluation of online interactive simulation software for learning human genetics. Elektrotechnik \& Informationstechnik (e\&i), 125(5), 190-196.

HOLZINGER, A.; Kickmeier-Rust, M. D.; Wassertheurer, S.; Hessinger, M. (2009). Learning performance with interactive simulations in medical education: Lessons learned from results of learning complex physiological models with the HAEMO dynamics SIMulator. Computer and Education, 52, 292-301.

KNEEBONE, R. (2003) Simulation in surgical training: educational issues and practical implications. Medical Education. 37(3):267-77.

MACHADO, L.; MORAES, R.; NUNES, F. (2009) Serious Games para Saúde e Treinamento Imersivo. Book Chapter. In: Fatima L. S. Nunes; Liliane S. Machado; 
Marcio S. Pinho; Claudio Kirner. (Org.). Abordagens Práticas de Realidade Virtual e Aumentada. Porto Alegre: SBC, 2009. p. 31-60

MACHADO, L.S. Zuffo, M.K. (2003) Development and Evaluation of a Simulator of Invasive Procedures in Pediatric Bone Marrow Transplant. Studies In Health Technology And Informatics. Amsterdam. v.94, p.193 - 195. USA

MARIS, J. J. N. et al. (org) (2004). Educação médica em transformação: instrumentos para a construção de novas realidades. São Paulo, Hucitec, 2004.

MORAIS, Alana Marques, et. al. Serious Games na Odontologia: Aplicações, Características e Possibilidades (2010) . In: XII Congresso Brasileiro de Informática em Saúde 2010. Porto de Galinhas/PE. Anais. 2010. CD-ROM.

PEARL, Judea. (1993) Belief Networks Revisited. Artificial Intelligence. Amsterdam: Elsevier, v.59, p.49-56, 1993.

PEARL, Judea. (1986) Fusion, Propagation, and Structuring in Belief Networks. Artificial Intelligence. North Holland, v.29, p.241-288, 1986.

PORTELA, M.C., ESCOSTEGUY, C.C., LIMA, S.M.L, FERREIRA, V.M.B, VASCONCELLOS, M.T.L, BRITO, C. (2008). Diretrizes Clínicas e outras práticas voltadas para a melhoria da qualidade assistencial em operadoras de planos de saúde sob a perspectiva de seus dirigentes no Brasil. Cad Saúde Pública 2008; 24:253-266.

RENDAS, A.; PINTO, P. R.; GAMBOA, T. A computer simulation designed for problem-based learning. Medical Education, 1999, 33, 047-054.

SCHWARTZ, A.; ELSTEIN, A. S. (2008). Clinical reasoning in medicine. In: Clinical Reasoning in the Health Professions. Elsevier. 3. Ed. 2008.

SEIXAS, Louise J.; FLORES, Cecília D.; GLUZ João C.; VICARI, Rosa M. Acompanhamento do processo de construção do conhecimento por meio de um agente probabilístico [online]. Via WWW. URL: http://saudecoletiva.ufcspa.edu.br/dflores/Publicacoes/AMPLIA_Sbie2004.PDF. Arquivo capturado em 27 de outubro de 2010.

SMITH, S. J.; Roehrs, C. J. (2009). High-fidelity simulation: Factor correlated with nursing student satisfaction and self-confidence. Nursing Education Perspectives, 30(2), 77-78.

STANFORD, P. G. (2010) Simulation in Nursing Education: a review of the research. The Qualitative Report. Nova Southeastern University - Florida - USA. v. 15, n. 14. Disponível em: www.nova.edi/ssss/QR/QR 15-4/stanford.pdf. Acesso em janeiro de 2012.

YING, L. S. (2009). Bringing "case" and learning "to life" using simulation technology. Singapore Nursing Journal, 36(3), 12, 14-16.

ZIV, A.; Ben-David, S.; Ziv, M. Simulation Based Medical Education: an opportunity to learn from errors. Medical Teacher, Vol. 27, No. 3, 2005, pp. 193-199. 